\title{
Initial Experience with Tricortical Iliac Crest Bone Graft and Human Amniotic Allograft in Evans Calcaneal Osteotomy
}

\author{
J. Joseph Anderson1, Adam F. Gough'2, Myron H. Hansen³ ${ }^{3}$, Zflan Swayzee1 \\ ${ }^{1}$ New Mexico Bone \& Joint Institute, Alamogordo, NM, USA \\ ${ }^{2}$ Silver Medicine Group, Silver City, NM, USA \\ ${ }^{3}$ Cactus Foot \& Ankle, Gilbert, AZ, USA \\ Email: jiosephanderson5@gmail.com
}

Received 25 March 2015; accepted 19 April 2015; published 22 April 2015

Copyright (C) 2015 by authors and Scientific Research Publishing Inc.

This work is licensed under the Creative Commons Attribution International License (CC BY). http://creativecommons.org/licenses/by/4.0/

(c) (i) Open Access

\begin{abstract}
Background: Adult acquired flatfoot deformity is generally mediated with an Evans procedure where a wedge of bone is placed into the calcaneus to better align the foot and decrease the deformity. The purpose of this study was to assess the efficacy and safety of human amniotic allograft applied to allogeneic tri-cortical grafts in Evans calcaneal osteotomy. Methods: The medical records of patients who had Evans calcaneal osteotomy with implantation of tri-cortical iliac crest bone graft with human anmiotic allograft for surgical management of adult acquired flatfoot deformity with 2 years follow-up data were reviewed. Results: A total of 63 patients (mean age: 33.3 yr, range: 18 - $66 \mathrm{yr}$ ) were enrolled with adult acquired flatfoot deformity. Median time to weightbearing was 6 weeks. Time to wearing normal shoes was 10 weeks, and time to radiographic healing was 16 weeks. Conclusions: The use of human amniotic allograft did not diminish the long term outcome of procedure or the short term benchmarks for healing after surgery. There were no nonunion, wound dehiscence, infection, or allergic or immune reaction reported. This retrospective study demonstrated that tri-cortical iliac crest bone graft and HAA could be safely used in Evans calcaneal osteotomy with favorable results.
\end{abstract}

\section{Keywords}

Adult Acquired Flatfoot Deformity, Bone Graft, Calcaneal Osteotomy, Evans Procedure, Human Amniotic Allograft

\section{Introduction}

This adult acquired flatfoot deformity (AAFD) presents a therapeutic challenge to foot and ankle specialists.

How to cite this paper: Anderson, J.J., Gough, A.F., Hansen, M.H. and Swayzee, Z. (2015) Initial Experience with Tricortical Iliac Crest Bone Graft and Human Amniotic Allograft in Evans Calcaneal Osteotomy. Stem Cell Discovery, 5, 11-17. 
Although $80 \%$ of patients with early AAFD respond well to nonoperative management, many patients with moderate or severe AAFD are best treated surgically. The Evans calcaneal osteotomy has become a primary surgical treatment for AAFD where a major portion of the deformity is in the transverse plane [1]. Iliac crest autograft is historically used to fill the calcaneal defect while performing an Evans procedure. However, donor site morbidity, delayed union, and prolonged time to full weight-wearing are limitations of this procedure [2]-[4].

A variety of bone graft substitutes have been utilized in order to overcome these limitations. Amniotic fluids and placental tissue have been used since early treatment of burn victims with applied human amnion to affected regions. Reports of this have come from the middle ages to even as recent as the early 19th century with fairly good results reported [5] [6]. The use of amnion-derived cells has been shown as a more reliable source of stem cells than bone marrow and embryonic and over the last few years has made advances in the fields of regenerative medicine and cellular therapy [7] [8]. The ability of the amniotic stem cells to differentiate into a variety of cell types allows it to be beneficial for modern grafts and tissues that can be applied to repair or replace a damaged structure or tissue in the body.

In 1938, the use of human amniotic cells (HAA) was reportedly used for instability of the lumbar spine. Originally, it was not known that there was a significant amount of disease and illness that could be transferred and also potential for both harvest and donor site application complications [9]. As recombinant DNA science and technology have advanced, there has been a plethora of orthobiologics on the market [10]-[14]. Surgeons have been searching for an optimal allograft to hold up to the measured gold standard of bone graft, which is human autograft [2] [15] [16]. A multitude of bone graft substitutes have been applied and used to avoid problems with donor site complications [17]. There have been no publications on complications having to do with amniotic stem cells. Given that these cells are a precursor line, it is felt that these amniotic stem cells would avoid the antigenicity and also have the benefit of being pluripotential and developing into local environment induced cell types to help facilitate allograft fusions [18]. The authors have taken this approach to the Evans calcaneal graft. There are reports of complications with Evans graft having to do with displacement of graft and also with nonunion of graft [2] [3]. There is also a significant amount of time for consolidation of a tri-cortical iliac crest bone graft as originally described by Evans [4]. The authors have been using human amniotic allograft (HAA) applied to allogeneic tri-cortical grafts in high-risk fusion sites and fractures in order to facilitate bony ingrowth and decrease healing times.

The purpose of this study was to assess the efficacy and safety of HAA applied to human tri-cortical allograft in Evans calcaneal osteotomies while correcting isolated transverse plane deformities. The authors hope that the use of human amniotic allograft in an Evans calcaneal osteotomy with a tri-cortical iliac crest bone graft will prevent non unions and mal unions and that complications will be low as well as improved healing times and rates.

There is a significant amount of time associated with incorporation and also time to full weight-bearing with flatfoot surgery, specifically related to calcaneal osteotomies [4]. The calcaneus is a well vascularized bone but the graft itself takes a significant amount of time to fully incorporate given the limitations of the surrounding cortical cancellous bone to fully grow across the applied tri-cortical graft at the osteotomy site. The authors undertook this study to evaluate the usefulness of HAA as related by healing. The surgeons have been using HAA applied to human tri-cortical allograft in difficult high-risk fusion sites and fractures in attempt to facilitate faster bony ingrowth, theoretically to facilitate better healing and decrease the healing time of patients [18].

\section{Methods}

\subsection{Chart Review}

The medical records and imaging studies of consecutive adult patients who had Evans calcaneal osteotomy with implantation of tri-cortical iliac crest bone graft with HAA for surgical management of AAFD from the practices of three authors (JJA, AFG and MHH) between January 2008 and October 2011 were reviewed. All of the included cases had 2 years of available follow-up data. Patients who underwent additional concomitant procedures, other than gastrocnemius recession, including bilateral osteotomy, or were diagnosed with any neuromuscular or connective tissue disorder, and patients with more extensive procedures were excluded. Graft incorporation and bone union were defined as the radiographic presence of trabeculation across the host-graft interface and was determined by blinded assessments from three investigators (JJA, AFG and MHH). Disagreements were settled 
by discussion and consensus.

\subsection{Surgical Procedure}

The patient was placed supine on the operating table with a bump placed beneath the ipsilateral buttock to medially rotate the foot. A pneumatic thigh tourniquet was typically used for hemostasis. An oblique or curvilinear incision was placed distal to the sinus tarsi and 1 to $1.5 \mathrm{~cm}$ proximal to the calcaneal-cuboid joint. An image intensifier was routinely used to confirm the osteotomy location prior to execution. Once satisfied with the location, the periosteum was incised in a vertical fashion, and an elevator was used to dissect periosteum from the lateral wall of the calcaneus. Peri-articular dissection was limited to avoid destabilization of the distal segment. A sagittal saw blade was oriented perpendicular to the lateral surface of the calcaneus and perpendicular to the weight-bearing surface to initiate the osteotomy in a lateral-to-medial direction. A handheld osteotome was typically used to complete the cut without violating the medial osseous hinge and soft tissue structures. A lamina spreader was used to manipulate and distract the osteotomy to the desired length to achieve restoration of arch height. The precise degree of correction was determined intraoperatively with fluoroscopy in conjunction with direct visualization of the sagittal, frontal, and transverse plane orientations of the foot (Figure 1).

The tricortical allograft was cut to the correct size and the liquid HAA graft was applied directly. This is left to soak for approximately 10 minutes while an endoscopic gastrocnemius recession is performed. The tricortical iliac/HAA graft is then applied to the osteotomy site.

The cortical base was oriented lateral and apex medial, and then tamped into its final position. The remaining HAA graft was applied to the surgical site prior to closure (Figure 2). K-wire fixation was used in $30 \%$ of cases. After application of a surgical dressing, a short-leg, non-weight-bearing cast was used and serial radiographs were obtained to determine the status of graft incorporation. When clinical and radiographic signs of healing were noted, the patient was transitioned to full weight bearing in a shortleg walker-boot that immobilized the ankle, after which a gradual transition to an athletic shoe was undertaken (Figure 3).

\subsection{Product Information}

Human amniotic allograft has been commercially available over the last decade. It is an allograft derived from human amnion and amniotic fluid. The properties are specifically beneficial and those that have been studied contain multipotential stem cells, multiple growth factors, atrophic proteins including bone morphogenic proteins, hyaluronic acid and collagen precursors of types III, IV and V and proteoglycans [19]. Because of cellular
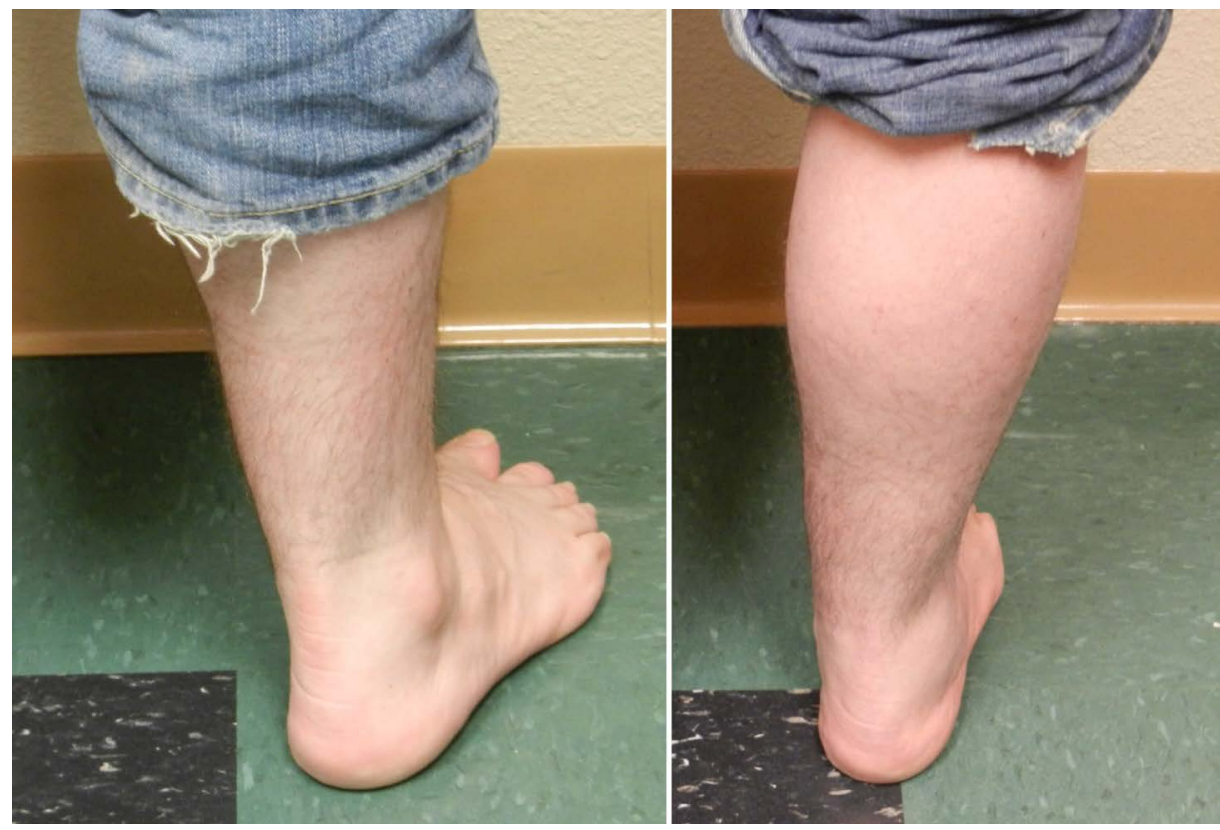

Figure 1. Patient ankle before and after Evans procedure with human amniotic allograft. 

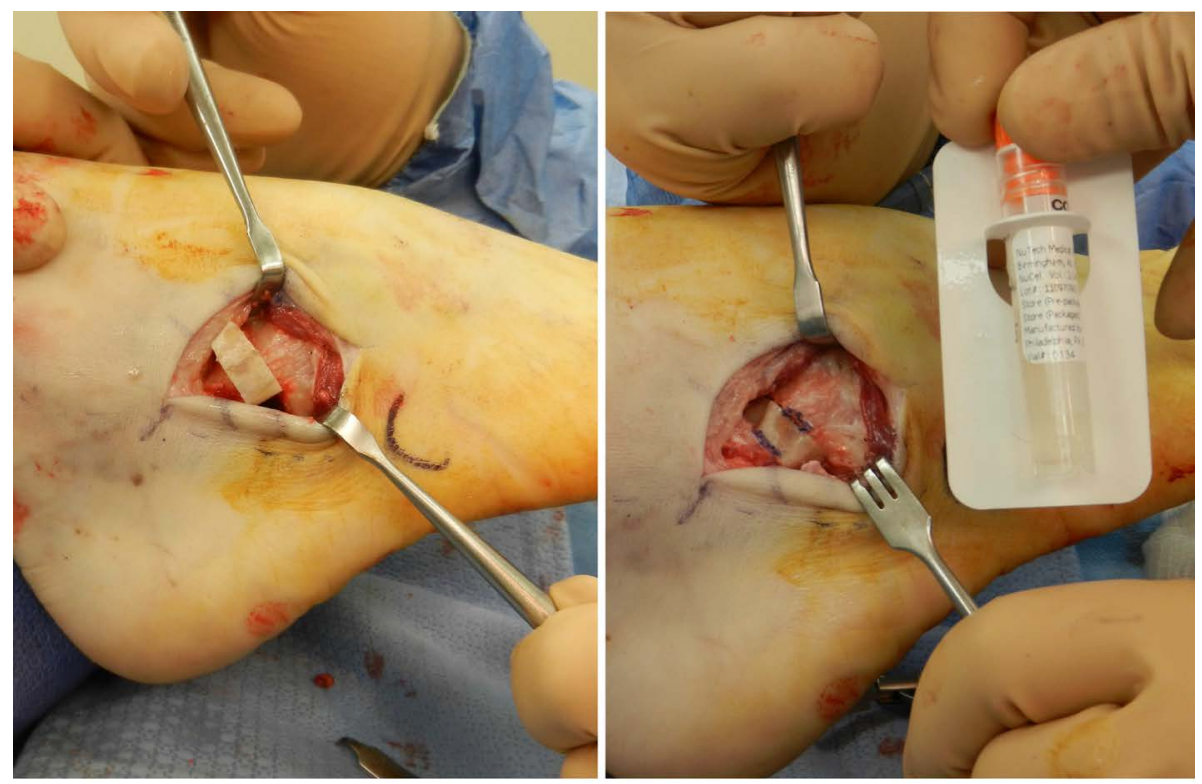

Figure 2. Patient intraoperative Evans procedure with liquid human amniotic allograft.

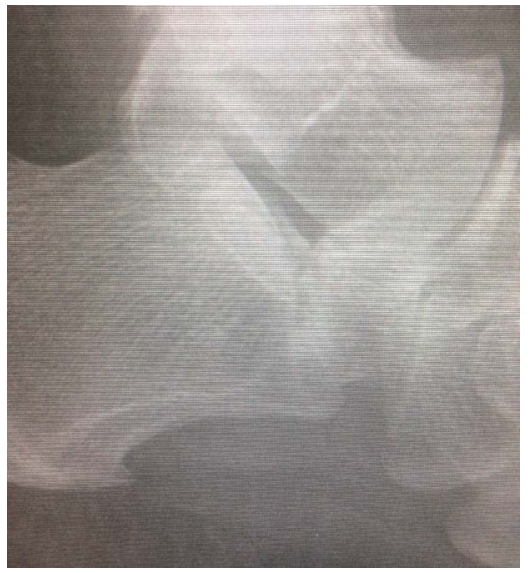

(a)

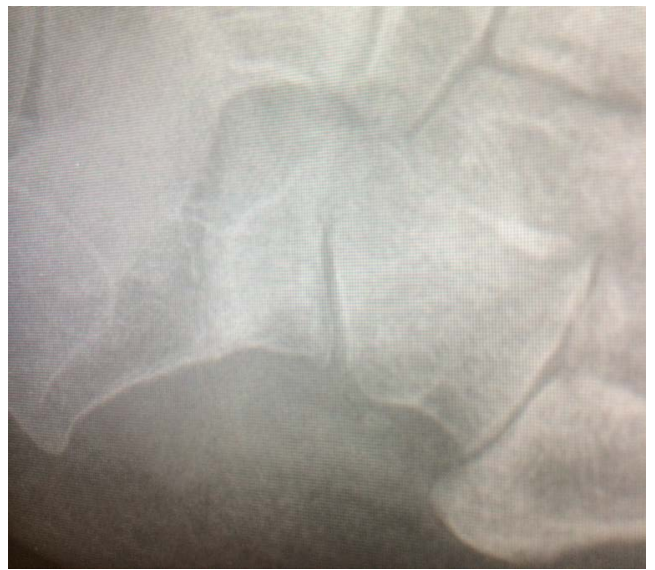

(b)

Figure 3. Evans procedure postoperative with (a) incomplete incorporation for graft at approximately 11 weeks and (b) complete incorporation of graft at approximately 14 weeks.

tissues being separated from amniotic fluid during the process of cultivating the human allograft, there is no antigenicity compared to fresh donor cells. The human allograft is tested via the standard donor testing for both antibodies to HIV, hepatitis B and C, HTLV-1 and HTLV-2 and other diseases as applicable to the specific processing. The patients are also screened individually prior to donation.

The product the authors used belongs to a new class of material derived from human amniotic tissue. It consists of fluid containing both morselized amniotic membrane and cells derived from amniotic fluid, all maintained in a cryopreserved state. The amniotic fluid and tissues are known to contain human collage, trophic proteins and hyaluronic acid along with multipotential cells of fetal origin [20]. Amniotic products have a long history of use in various medical applications. Human amniotic tissue allografts are not known to cause any significant immune response on implantation in a multitude of areas [5]. Human amniotic allografts have also demonstrated bioactivity in multiple studies [21]-[23].

\subsection{Data Analysis}

Main outcomes included time to weight bearing, time to wearing normal shoes and time to radiographic healing. 
Continuous data were reported as mean \pm SD or median and range, depending on normality assumptions. Categorical data were reported as frequencies and percentages. Longitudinal changes in clinical outcomes were assessed with repeated measures analysis of variance. A p-value $<0.05$ was considered statistically significant and performed with student t-test. Data were analyzed using SPSS v. 22 (IBM Inc., Armonk, NY).

\section{Results}

There were 63 patients undergoing the Evans procedure with HAA. Age and sex between the patients didn't provide any statistical significance. The average for weight-bearing, time into shoes and radiographic healing were, in weeks, 6.12, 10.07 and 16.21 respectively. They were not significantly different when compared to the normal range of healing after an Evans calcaneal osteotomy and tri-cortical iliac crest bone graft of about 6 weeks to weight-bearing, 10-12 weeks for time in shoes and 12 - 16 weeks for radiographic healing. The use of a Kirschner wire to fixate had no significant effect on the outcomes of the times to weight-bearing, wearing shoes, and radiographic healing, or complications ( $p=0.40, p=0.44, p=0.46, p=0.24$ respectively).

All patients, except 1 , admitted that they would have the procedure again. There were no wound dehiscence and no infections of any kind post-operatively ( $0 \%$ and $0 \%$, respectively). One patient required some correction at 3 weeks due to a thin iliac graft that was found intra-operatively and another needed a sub-talar joint fusion 1 year after the initial Evans procedure(1.6\% and 1.6\%, respectively), but the overall complication rates were not significant $(\mathrm{p}=0.16)$. It should be noted that there were no cases within the study group that had an allergic or immune reaction. There were no other profound or significant complications.

\section{Discussion}

This retrospective study demonstrated that tri-cortical iliac crest bone graft and HAA can be safely used in Evans calcaneal osteotomy with favorable results. Although the independent effect of HAA above that of tricortical allograft is currently unknown, the fact that we observed no graft-related complications and durable clinical improvements through 2 years is promising.

Amniotic membrane is a versatile, safe and effective treatment for a variety of wounds and presents a low risk of immunologic rejection [24]-[26]. Decades of published clinical experience support the conclusion that human amnion can have favorable effects on wound healing [27]-[32]. The orthopedic application of amniotic fluid dates back to the 1930s. The bioactivity and positive effects of amniochorionic membranes and amniotic fluid have been reported to have beneficial effects on bone fusion and fracture healing [7] [33]-[35]. These prior reports are consistent with our findings, which showed no infection, graft rejection, or non-union in patients undergoing Evans calcaneal osteotomy. Additionally, our experience in this series was that liquid HAA handles easily in a syringe and easily thawed in warm water after removal from the bone freezer, stored at $-40^{\circ} \mathrm{F}$.

This is the first report of HAA in Evans calcaneal osteotomy. However, there are several limitations of this study worth mention including the retrospective design and lack of a control group. Nonetheless, the results reported herein are promising and the authors are continuing their investigations concerning the human amniotic allograft.

\section{References}

[1] Green, D.R. and Carol, A. (1984) Planal Dominance. Journal of the American Podiatric Medical Association, 74, 98103. http://dx.doi.org/10.7547/87507315-74-2-98

[2] Parikh, S.N. (2002) Bone Graft Substitutes: Past, Present, Future. Journal of Postgraduate Medicine, 48, 142-148.

[3] Miki, T. and Strom, S.C. (2006) Amnion-Derived Pluripotent/Multipotent Stem Cells. Stem Cell Reviews, 2, $133-141$. http://dx.doi.org/10.7547/87507315-74-2-98

[4] Evans, D.L. (1953) Recurrent Instability of the Ankle-A Method of Surgical Treatment. Proceedings of the Royal Society of Medicine, 46, 343-344.

[5] Hatch, E.L. (1999) Burns and Bravery. In: Hatch, E.L., Ed., Médico: My Life as a Country Doctor in Mexico, 193-195.

[6] Gwei-Djen, L. and Needham, J. (1964) Medieval Preparations of Urinary Steroid Hormones. Medical History, 8, $101-121$. http://dx.doi.org/10.1017/S0025727300029355

[7] Insausti, C.L., Blanquer, M., Bleda, P., Iniesta, P., Majado, M.J., Castellanos, G. and Moraleda, M. (2010) The Amniotic Membrane as a Source of Stem Cells. Histology and Histopathology, 25, 91-98. 
[8] Saito, S., Lin, Y.C., Murayama, Y., Hashimoto, K. and Yokoyama, K.K. (2012) Human Amnion-Derived Cells as a Reliable Source of Stem Cells. Current Molecular Medicine, 12, 1340-1349. http://dx.doi.org/10.2174/156652412803833625

[9] Shimberg, M. (1938) The Use of Amniotic-Fluid Concentrate in Orthopaedic Conditions. Journal of Bone and Joint Surgery, 20, 167-177.

[10] Anderson, J.J. (2009) Bone Grafting and Orthobiologics for Reconstruction of the Diabetic Lower Extremity. In: Zgonis, T., Ed., Surgical Reconstruction of the Diabetic Foot and Ankle, Philadelphia, 192-193.

[11] Cornell, C.N. (1999) Osteoconductive Materials and Their Role as Substitutes for Autogenous Bone Grafts. Orthopedic Clinics of North America, 30, 591-598. http://dx.doi.org/10.1016/S0030-5898(05)70112-7

[12] Weinraub, G.M. (2005) Orthobiologics: A Survey of Materials and Techniques. Clinics in Podiatric Medicine and Surgery, 22, 509-519. http://dx.doi.org/10.1016/j.cpm.2005.08.003

[13] Lane, J.M. and Bromstrom, M.P.G. (1998) Bone Grafting and New Composite Biosynthestic Graft Materials. AAOS Instructional Course Lectures, 47, 525-534.

[14] Martin, R.B., Chapman, M.W., Sharkey, N.A., Zissimos, S.L., Bay, B. and Shors, E.G. (1993) Bone Ingrowth and Mechanical Properties of Coralline Hydroxyapatite 1 Year from Implantation. Biomaterials, 14, 341-348. http://dx.doi.org/10.1016/0142-9612(93)90052-4

[15] Anderson, J.J., Boone, J.J., Hansen, M., Brady, C., Gough, A. and Swayzee, Z. (2014) Ankle Arthrodesis Fusion Rates for Mesenchymal Stem Cell Bone Allograft verses Proxiamal Tibia Autograft. Journal of Foot and Ankle Surgery, 53, 683-686. http://dx.doi.org/10.1053/j.jfas.2014.06.029

[16] Anderson, J.J., Jeppesen, N.S., Hansen, M., Brady, C. and Gough, A. (2013) First Metatarsophalangeal Joint Arthrodesis: Comparison of Mesenchymal Stem Cell Allograft versus Autogenous Bone Graft Fusion Rates. Surgical Science, 4, 251-287. http://dx.doi.org/10.4236/ss.2013.45051

[17] Mahan, K.T. (2004) Complications of Evans Calcaneal Osteotomy. Podiatr Inst Update 2004.

[18] Anderson, J.J., Hansen, M.H. and Swayzee, Z. (2014) Human Amniotic Allograft in Use on Talar Dome Lesions: A Prospective Report of 37 Patients. Stem Cell Injection, 5, 55-60. http://dx.doi.org/10.4236/scd.2014.43006

[19] Davitt, J.S., MacWilliams, B.A. and Armstrong, P.F. (2001) Plantar Pressure and Radiographic Changes after Distal Calcaneal Legthening in Children and Adolescents. Journal of Pediatric Orthopaedics, 21, 70-75. http://dx.doi.org/10.1097/01241398-200101000-00015

[20] NuTech Medical (2012) NuCel: Multipotential Cellular Matrix (HCT/P) 1, 1-2.

[21] NuTech Medical (2012) NuCel: Product Overview. 1, 1 -2.

[22] Sato, T.A., Keelan, J.A. and Mitchel, M.D. (2003) Critical Paracrine Interactions between TNF- $\alpha$ and IL-10 Regulate Lipopolysaccharide-Stimulated Human Choriodecidual Cytokine and Prostaglandin E2 Production. The Journal of Immunology, 170, 158-166. http://dx.doi.org/10.4049/jimmunol.170.1.158

[23] Guldberg, R.E., Oest, M.E., Dupont, K., Peister, A., Deutsch, E., Kolambkar, Y. and Mooney, D. (2007) Biologic Augmentation of Polymer Scaffolds for Bone Repair. Journal of Musculoskeletal and Neuronal Interactions, 7, 333-334.

[24] Davitt, J.S., MacWilliams, B.A. and Armstrong, P.F. (2001) Plantar Pressure and Radiographic Changes after Distal Calcaneal Lengthening in Children and Adolescents. Journal of Pediatric Orthopaedics, 21, 70-75. http://dx.doi.org/10.1097/01241398-200101000-00015

[25] Faulk, W.P., Matthews, R., Stevens, P.J., Bennett, J.P., Burgos, H. and His, B.L. (1980) Human Amnion as an Adjunct in Wound Healing. Lancet, 1, 1156-1158. http://dx.doi.org/10.1016/S0140-6736(80)91617-7

[26] Akle, C.A., Adinolfi, M., Welsh, K.I., Leibowitz, S. and McColl, I. (1981) Immunogenicity of Human Amniotic Epithelial Cells after Transplantation into Volunteers. Lancet, 2, 1003-1005. http://dx.doi.org/10.1016/S0140-6736(81)91212-5

[27] Shukla, V.K., Rasheed, M.A., Kumar, M., Gupta, S.K. and Pandey, S.S. (2004) A Trial to Determine the Role of Placental Extract in the Treatment of Chronic Non-Healing Wounds. Journal of Wound Care, 13, 177-179. http://dx.doi.org/10.12968/jowc.2004.13.5.26668

[28] Cole, F.R. (1948) Placental Blood and Placental Extract in Wound Healing. The American Journal of Surgery, 76, 3843. http://dx.doi.org/10.1016/S0002-9610(48)90182-2

[29] Pigeon, J. (1960) Treatment of Second-Degree Burns with Amniotic Membranes. Canadian Medical Association Journal, 83, 844-845.

[30] Trelford, J.D. and Trelford-Sauder, M. (1979) The Amnion in Surgery, Past and Present. American Journal of Obstetrics \& Gynecology, 134, 833-845.

[31] Quinby Jr., W.C., Hoover, H.C., Scheflan, M., Walters, P.T., Slavin, S.A. and Bondoc, C.C. (1982) Clinical Trials of 
Amniotic Membranes in Burn Wound Care. Plastic and Reconstructive Surgery, 70, 711-717. http://dx.doi.org/10.1097/00006534-198212000-00009

[32] Ward, D.J., Bennett, J.P., Burgos, H. and Fabre, J. (1989) The Healing of Chronic Venous Leg Ulcers with Prepared Human Amnion. British Journal of Plastic Surgery, 42, 463-467. http://dx.doi.org/10.1016/0007-1226(89)90015-5

[33] Mermet, I., Pottier, N., Sainthillier, J.M., Malugani, C., Cairey-Remonnay, S., Maddens, S., Riethmuller, D., Tiberghien, P., Humbert, P. and Aubin, F. (2007) Use of Amniotic Membrane Transplantation in the Treatment of Venous Leg Ulcers. Wound Repair and Regeneration, 15, 459-464. http://dx.doi.org/10.1111/j.1524-475X.2007.00252.x

[34] Karacal, N., Kosucu, P., Cobanglu, U. and Kutlu, N. (2005) Effect of Human Amniotic Fluid on Bone Healing. Journal of Surgical Research, 129, 283-287. http://dx.doi.org/10.1016/j.jss.2005.03.026

[35] Kerimoglu, S., Livaoglu, M., Sonmez, B., Yulug, E., Aynaci, O., Topbas, M. and Yarar, S. (2009) Effects of Human Amniotic Fluid on Fracture Healing in Rat Tibia. Journal of Surgical Research, 152, 281-287. http://dx.doi.org/10.1016/j.jss.2008.02.028 